\title{
Importancia de las nuevas tendencias de marketing en las PYMES
}

\author{
Núñez, Byron \\ Escuela Superior Politécnica de Chimborazo, Riobamba, Ecuador \\ E-mail: byronnuvi@hotmail.com
}

\author{
Recibido: 22 mayo de 2019 \\ Aprobado: 23 diciembre de 2019
}

\begin{abstract}
Resumen
Las PYMES en el mundo son el motor productor principal de un país, en Ecuador se diría que son la esperanza para un cambio de la matriz productiva. Lo particular de estas empresas es que ofertan sus productos mediante el marketing tradicional, a través de folletos, afiches, etc. que probablemente muchos de estos terminaran en la basura, otros utilizaran la radio o TV, pero son costosos y no todos tienen la posibilidad de gastar tal cantidad de dinero y el objetivo que se quiere obtener con esto, es llegar a un gran número de personas, en realidad solo alcanzará a un pequeño porcentaje, es importante diferenciar entre marketing y publicidad, el marketing expresa una estrategia general para atraer clientes, la publicidad hace referencia a la comunicación, y a la presentación o promoción de los productos a los clientes existentes y potenciales. Además que con estrategias ingeniosas sobresalen para ser cada vez más competitivos, sin este instrumento muy probablemente están condenadas al fracaso. El autor cree que se debe concientizar a los propietarios para que salgan de su área de confort, ya que en su mayoría el objetivo es crear una manutención que cubra simplemente los gastos básicos de techo y alimentación, y la empresa que crearon es sin ninguna meta de crecimiento, sin una visión emprendedora. En la actualidad el estándar gold es el marketing online, a través de las redes sociales, que ha tomado fuerza y debería aprovechar este medio las PYMES, siendo una manera excelente de captar nuevos clientes o fidelizar los existentes. Las opciones son muy amplias, fácilmente localizables en la red y muchos de ellos gratuitos convirtiéndose en una ventaja, permitiendo promocionar y vender sus productos, las empresas crecerían y sin mayor inversión.
\end{abstract}

Palabras Clave: PYMES, Marketing, Tendencias, Fidelizar, Estrategia. 


\title{
Importance of new marketing trends in SMEs
}

\begin{abstract}
Around the world the PYMES are the main productive engines in the countries. In Ecuador hopefully PYMES will be the ones that will change the production matrix. The peculiarity in this companies is that they offer their products through traditional marketing with brochures, posters, etc. That probably many of them will finish in the trash. Others companies will use radio or T.V. advertisements, but those are expensive. And no of them are able to spend that amount of money. However, the objective is to captivate a big quantity of people. But at the end, the companies will reach a small percentage of them. It is important to make a difference between marketing and publicity. The marketing shows a general strategy to attract clients. On the other hand, publicity focus to communication and to promote products to actual and potential customers. Also with smart strategies they are more competitive every time. Because without this tool they will probably destined to fail. The author suggest awareness to the owners to leave their comfort zones. PYMES often focus just in how to paid expenses and how to maintain the company stable but they do not think about how to improve the development of the company. Nowadays the gold standard is the online marketing by the social networks that became powerful the last years, because it is a good way to find new costumers and also to keep in contact with the old ones. It is a lot of options, easy to find and most of them are free, making it in an advantage. Because it allows to promote and sell products. This will make the companies growth without big investments.
\end{abstract}

Keywords: PYMES, Marketing, Trend topics, Keep contact, Strategy.

\section{Introducción}

Urbano, D. y Toledano N. (2008), "Las PYMES se definen comúnmente en función de: (1) el número de empleados, (2) el volumen anual de negocios, y (3) el balance anual.” (p.21) de acuerdo a este autor lo que propone es que las pymes son entidades que más allá de su forma jurídica, son un conjunto de recursos humanos, técnicos y materiales, dirigidos por una o varias personas que tomando decisiones oportunas, logran generar utilidades.

Alcaide, J. (2013), en su libro mencionan que el panorama actual ha cambiado radicalmente al que era hace unos pocos años. Antes en el plan de marketing se incluía medios y acciones ejecutadas en un mundo real. El éxito de este plan en gran parte dependía de los medios utilizados y de la inversión. Hoy es completamente distinto, se tiene una oportunidad muy grande en el mundo digital.

El Internet es el nuevo ecosistema repleto de oportunidades y herramientas que sin necesidad de tener grandes recursos económicos va ayudar tanto a la empresa pequeña como grande a anunciar sus productos, marcas, características, etc. Tan solo haría falta la creatividad, planificación y constancia.

Ya sean las empresas grandes o pequeñas no pueden escaparse del salto gigantesco que han dado las nuevas tecnologías de comunicación, que básicamente serían las redes sociales a través del internet, esto puede ser usado favorablemente el autor piensa que el potencial del marketing digital ha sido aprovechado en una mínima parte. Como dice Aulestia, J. (2013), en su Revista Perspectiva, "Puede ser que en los últimos 10 años tal evolución tecnológica ha crecido tanto sin pedir permiso alguno y por tal razón no se ha visto una real necesidad de uso en la PYMES."(p.27)

Dando lugar a una necesidad de investigación del estado actual de las redes sociales como medios de comunicación para

Núñez, Byron 
las PYMES, llegando de una forma masiva al consumidor o clientes.

Sabiendo que las pequeñas y medianas empresas pueden llegar a ser la base fundamental para un cambio drástico en la matriz productiva del Ecuador, esto es un llamado a ser más creativos y eficientes al momento de dar a conocer los productos para seguir captando mercado de tal forma que las empresas sean sostenibles en el tiempo y puedan generar más fuentes de empleo.

Alcaide, J. y otros (2013), en su libro lo mencionan, no es suficiente solo tener una página web ya que no ha sido diseñada con una versión marketiniana y tomando en cuenta el SEO (Search Engine Optimization) la optimización de una web.

Hoy por hoy en el mundo cibernético la mejor herramienta que la PYMES puede tener para promocionar sus productos, servicios y aumentar sus ventas es el Internet. Siendo el punto de partida para que llegue la información de manera efectiva a las personas interesadas, teniendo de esta forma una vía de marketing más eficiente y menos costoso.

\section{Desarrollo}

Kotler, P. (2001), el marketing es: "el proceso social y gerencial por el cual individuos $\mathrm{y}$ grupos obtienen lo que necesitan y desean mediante la creación e intercambio de productos y valor con otros" de acuerdo a este autor esto es lo que se ha venido haciendo a lo largo de la historia, pero visiblemente los tiempos cambian por ello la importancia de aplicar las tendencias del marketing con la tecnología en si con el internet. Ya que a través de este medio da la oportunidad de segmentar mercados, conocer mejor a los clientes para poder fidelizarlos.

Ros, V. (2008), "en el mundo on-line, las marcas deben ser conscientes y confiar en la importancia de las nuevas formas publicitarias como vehículos para aumentar la eficiencia de sus esfuerzos de marketing", siendo esta una muy buena alternativa para las PYMES para poder impactar en los consumidores de una forma eficaz.

El desarrollo de diversas plataformas abre una puerta grande para poder aplicar el concepto de marketing y lo más probable a costos más bajos que los medios convencionales, radio, televisión, entre otros.

Hay que tomar en cuenta los factores críticos en el desarrollo de las Pymes, ya que por su nivel bajo de ingresos e inversión modesta, están sujetas a la quiebra debido a muchas percances que confrontan a la hora de competir.

Moral, M. y Fernández M. (2012), En su artículo indica que "el incremento de la competencia en los mercados y la existencia de una mayor competitividad entre las empresas, así como los cambios en el comportamiento de compra de los consumidores ha puesto de manifiesto la necesidad de adoptar una concepción del marketing más adaptada a la realidad actual del mercado y de las demandas de los consumidores" de acuerdo a este autor esta realidad hace que las empresas tengan nuevas oportunidades de negocios en las diferentes plataformas informáticas y redes sociales.

Es por ello que tomando en cuenta la situación real de las PYMES, que no siempre 
cuentan con un presupuesto alto para sus estrategias de ventas, hay que buscar métodos en las que pueda desarrollarse, crecer y sobre todo ser competitivas otorgando al consumidor productos de calidad que lo satisfaga, y una respuesta positiva a esto como vía fácil de acceso por las nuevas tendencias de comunicación que hay en la actualidad, el autor considera que puede ser el Internet, siendo un medio muy usado por los clientes para adherirse al mercado de bienes y servicios, siendo una de las herramientas de mayor dominio en la actualidad por un nicho segmentado de clientes, permitiendo elegir lo que quieren leer, escuchar, y con ello limitar el marketing tradicional, cumpliendo así dos objetivos, desechar la información que no interesa e ingresar a la información que se considere relevante para adquirir algún producto, bien o servicio que lo requiera, dando como resultado un acceso fácil sin limitación geográfica.

Moral, M. y Fernández M. (2012), También cita en su artículo que el "marketing experiencial, uno de los últimos avances de la disciplina del Marketing, que se dirige a ofrecer al consumidor una experiencia de consumo única, estableciendo una vinculación con la marca o empresa, basada en las emociones, sentimientos o pensamientos, entre otros aspectos, que despierta el producto en el consumidor", de acuerdo con este autor se puede lograr con el cliente una fidelización entre la empresa o marca, incrementando así las ventas de los productos de la empresa por ende incrementado sus utilidades.

Rodríguez, T. (2013), en su artículo menciona que, el uso que los clientes le den a cada plataforma va delinear las funciones de las redes sociales.

En este entorno virtual, las pymes pueden ser competitivas con las grandes empresas. El manejo adecuado y creativo de las redes sociales radica en la concienciación por parte de las pymes, ya que es la imagen de la empresa la que está en la web, de la que se habla, opina, comenta, hace referencia. Cualquier comentario no atendido o contestado de una manera no profesional puede generar reputación negativa sobre la marca y deteriorar muchos años de gestión empresarial off line de la empresa.

El marketing online, o digital, ha evolucionado mucho al marketing social, combinando las premisas del marketing digital y el marketing directo, teniendo como objetivo mejorar las comunicaciones en lo que respecta a responsabilidad social empresarial, generar interacción social como también mostrar interés comercial por los requerimientos del consumidor.

Rodríguez (2013), en su artículo indica la comunicación que las empresas tengan con su mercado meta-objetivo puede lograrse, alcanzando los objetivos corporativos. Un eje transversal para la gestión de las redes sociales es la aprehensión, por parte de las organizaciones de estilos comunicacionales basados en escuchas activas de sus públicos (p.32) por lo que se puede establecer la importancia de ser creativos al momento de plasmar la información de los artículos o marcas de la pymes, seleccionando adecuadamente cada contenido que va ir en las plataformas informáticas o redes sociales de tal manera que se lo pueda hacer muy atractivo para el consumidor.

Valdez, L. y otros (2012), En un entorno 
de máxima competitividad empresarial, resulta fundamental para cualquier organización tomar las medidas estrategias que le permitan obtener mejores resultados en su gestión de negocios. La aplicación de tecnologías de información, específicamente las herramientas de comunicación como redes sociales se han convertido en una excelente oportunidad para las pequeñas y medianas empresas en su afán de tener más presencia de mercado (p.32) este autor basado en la accesibilidad a la plataformas, ya que las zonas Wifi y los planes de datos están cada vez más al alcance de los usuarios pueden visitar las páginas en los smartphones y tablets. Por ello se verá más integración de contenidos en dispositivos móviles. Para las PYMES. Ya no es suficiente tener una página web, sus contenidos deben estar accesibles en los diferentes dispositivos de lo contrario quedaran fuera de un importante porcentaje de mercado.

Incrementar la publicidad en la red social Facebook, siendo esta una de las redes sociales más usadas en Latinoamérica. Para las PYMES, puede ser ventajoso por presentar una segmentación de sus usuarios. Esto llevaría a que se debe aprender a vender a nichos específicos y no por tratar de abarcar a todos, se correría el riesgo de no llegar de forma óptima.

El marketing digital nos invita a estar un paso más adelante averiguando nuevos usos y recursos que por supuesto que lo haga más novedoso, la gran mayoría de empresas le apuesta al marketing de contenido siendo esta una de las más productivas y rentables, así los usuarios estarán informados referente a la marca, producto, precio, etc.
En el marketing de contenidos se puede mencionar que es de mucha importancia en vista que la generación de la información debe ser más creativa, debido que las horas promedio de una persona que pasa en los buscadores siguen siendo mayor. Definitivamente las PYMES deben tener estrategias muy innovadoras al momento de expresar los contenidos, descripciones de sus productos.

Ros, V. (2008), DoubleClick realizó un estudio sobre la efectividad del marketing on-line, que demuestra que este tipo de marketing resulta eficaz para la creación de marca en Internet. Según el estudio, a través de la publicidad on-line los mensajes de la marca llegan a impactar de forma eficaz en los consumidores (p.47), este autor lo que nos indica con este estudio es la efectividad que tienen estos medios cibernautas para poder influir en los clientes en la recordación de la marca o delos productos, debido al uso masivo de los dispositivos móviles.

Bongiovanni, M. (2005), en su artículo indica que "El Marketing cultural está dentro de estas nuevas formas de comunicación con que cuenta la empresa para dar a conocer su proyecto vital y su compromiso social" (p.8), este autor hace mención que para fortalecer la imagen de la empresa realiza un conjunto de actividades culturales y artísticas planificadas y organizadas, para que la ciudadanía perciba que la empresa esta como un objeto socialmente responsable.

El autor piensa que este estilo de marketing para una PYME de bajo presupuesto no es muy recomendable debido a que puede ocasionar una inversión alta.

Para las PYMES se puede indicar que 
incursionar en las nuevas tendencias de marketing es una buena alternativa para poder promocionar la empresa, la marca o los productos de una manera eficiente, ya que el uso de las diversas plataformas informáticas y redes sociales cada vez va aumentando su uso.

Con creatividad y un manejo adecuado del marketing de contenidos se puede crear en los clientes una expectativa interesante de tal manera que los clientes al momento de navegar o revisar la información de las PYMES lo hagan de una manera interactiva y lo principal el objetivo de lograr la recordación de marca o de producto sea efectiva.

Sobre todo esto se puede lograr con un presupuesto adecuado ya que es de bajo costo en relación a los del marketing tradicional.

\section{Conclusiones}

Se concluye que al usar las diferentes plataformas informáticas y redes sociales, debido al bajo presupuesto que tienen las PYMES para promocionar sus productos es de mucha importancia que se afiance como estrategia para vender de manera eficiente y poder segmentar a sus clientes. El marketing on-line para que pueda tener un alto impacto la publicidad en las redes sociales, hay que saber enfocar bien en cual red social la PYME debe anunciarse, sabiendo que la red social de una manera u otra segmenta el mercado.

Siendo creativos en el marketing de contenidos puede llegar a ser muy interesante para los usuarios de las diferentes plataformas informáticas enterarse de lo que las PYMES pueden ofertar, interactuar directamente con la empresa y poder fidelizar a los clientes. El Internet es el acceso más fácil y viable para que las PYMES entren al mundo online, hay que tener determinación y comenzar a utilizarlo, obteniendo beneficios para las empresas, como: recordación de marca o productos, información directa del consumidor para tener un feed back y poder tomar correctivos inmediatamente.

Aprovechar la tecnología, información y el conocimiento logra crear estrategias junto de un adecuado direccionamiento acceden a mejores ganancias para las PYMES. Las empresas tienen que estar dentro de un mejoramiento continuo en cuanto a canales de comunicación se refiere con sus clientes a través de las redes sociales $\mathrm{y}$ estar siempre acorde a sus necesidades $\mathrm{y}$ requerimientos de la sociedad moderna en la que nos encontramos, y que es exigente a la vez.

Los clientes al buscar "facilidad" en la adquisición de servicios y/o productos lo hacen a través de la vía online, esto obliga a que se incremente el uso de servicios, obviamente es más rápido y fácil, en el caso de las PYMES usar estos canales optimizará recursos y estará más cerca del cliente, además que el servicio será más rápido y eficiente, teniendo la posibilidad de abarcar más mercados y no quedarse fuera de la nueva tendencia cibernauta.

Al crecer la audiencia y por ende crece el alcance por parte de las marcas, cada vez se ve más tiendas usando los canales online claro está de una manera profesional e integral como lo es la compra y post venta online. Con las PYMES las compras Online se incrementarán y a su vez ira creciendo el E-Commerce, que ampliaran su oferta y su mismo mercado. 


\section{Referencias bibliográficas}

Alcaide, J. C., Bernués, S., Díaz-Aroca, E., Espinosa, R., Muñiz, R., \& Smith, C. (2013). Marketing y Pymes, Las principales claves de marketing en la pequeña y mediana empresa. Marketing Y Pymes.

Bongiovanni, M. P. (2005). Marketing cultural y responsabilidad social empresarial.

Bosh, V. (2014). Nuevas tendencias en el marketing.

Córdova, J. (2009). Del marketing transaccional al marketing relacional.

Llano, F. \& Calvo, J. (2007). Hoy es marketing, España, $\mathrm{PG}$

Merino, M. \& Yaguez, E. (2012). Nuevas tendencias en investigacion y marketing, España, PG

Moral, m. y Fernández M. (2012). Nuevas tendencias del marketing: el marketing experimental.

Reyes, O. (2013). Nuevas tendencias en el negocio electronico, EE UU, PG

Urbano, D. \& Toledano, N. (2008). Invitacion al emprendimiento.

Vertice. (2008). Marketing para pymes, España, PG 